\title{
VARIABILIDADE ESPACIAL DE PROPRIEDADES FÍSICAS DO SOLO EM UMA PARCELA EXPERIMENTAL (1)
}

\author{
Celia Regina Grego(2) \& Sidney Rosa Vieira ${ }^{(3)}$
}

\begin{abstract}
RESUMO
Experimentos de solo realizados no campo necessitam de estudos que verifiquem a variabilidade espacial do solo. $O$ objetivo deste trabalho foi estudar a variabilidade espacial de propriedades físico-hídricas do solo em uma parcela experimental, usando métodos geoestatísticos. 0 experimento foi realizado em 1982 no Centro Experimental do Instituto Agronômico em Campinas (SP), em um Latossolo Vermelho sob preparo convencional, numa parcela de $30 \times 30 \mathrm{~m}$, com grade de pontos a cada $5 \mathrm{~m}$. As propriedades analisadas foram: teor de água do solo, porosidade, densidade do solo, resistência à penetração e retenção de água. Para analisar a variabilidade espacial, utilizou-se a geoestatística, por meio da análise de semivariogramas, interpolação dos dados por krigagem e construção de mapas de isolinhas. A dependência espacial ocorreu principalmente nas variáveis obtidas na camada superficial do solo $(0-25 \mathrm{~cm})$, apresentando dependência espacial moderada e forte. Houve correlação positiva significativa entre retenção de água e densidade do solo. A dependência espacial encontrada e a semelhança de comportamento entre as variáveis permitiram inferir que amostragem ao acaso seria falha, pois esconderia a variabilidade encontrada, interferindo nas respostas dos tratamentos, caso fosse instalado um experimento que exigisse independência entre amostras.
\end{abstract}

Termos de indexação: semivariograma, krigagem, preparo convencional, Latossolo Vermelho.

\footnotetext{
(1) Recebido para publicação em dezembro de 2003 e aprovado em janeiro de 2005.

(2) Récem Doutotoranda do I nstituto Agronômico - IAC. Caixa Postal 28, CEP 13001-970 Campinas (SP). Bolsista do CNPq. E-mail: crgrego@iac.sp.gov.br

(3) Pesquisador do Centro de Solos e Recursos Agroambientais, IAC. E-mail: sidney@iac.sp.gov.br
} 


\title{
SUMMARY: SPATIAL VARIABILITY OF SOIL PHYSICAL PROPERTIES ON AN EXPERIMENTAL PLOT
}

\begin{abstract}
Field experiments involving soils require previous verification of the soil spatial variability. Theobjective of this research was to study thespatial variability of soil physical properties of an experimental plot using geostatistics. The experiment was conducted in 1982 at the Experimental Center of the Instituto Agronômico in Campinas, state of São Paulo, Brazil, on a Red Latosol (Rhodic Ustox) under conventional tillage in a $30 \times 30 \mathrm{~m}$ area, with sampling points arranged in a $5 \mathrm{~m}$ square grid. The analyzed properties were water content, porosity, bulk density, penetration resistance, and water retention. Thespatial variability was evaluated by geostatistical analysis of semivariograms and kriging interpolation of thedata for theconstruction of maps. Spatial dependenceoccurred mainly for the variables from the upper soil layer $(0-25 \mathrm{~cm})$, showing moderate and strong spatial dependence. A significant positivecorrelation was found betwen water retention and bulk density. The observed spatial dependenceand similar behavior of thevariables al lowed the inferencethat random sampl ing would not beenough to characterizea field, as it would fail to show the variability. In cases where an experiment requires independent samples, the response to the treatments would be affected.
\end{abstract}

Index terms: semivariogram, kriging, conventional tillage, Red Latosol .

\section{NTRODUÇÃO}

A aplicação detecnol ogia associada à variabilidade espacial etemporal faz-se necessária, sobretudo na pesquisa agrícola que estuda o solo e a sua capacidade produtiva. O conhecimento da variabilidade das propriedades dosolo edas culturas, no espaço e no tempo, é considerado, atual mente, o princípio básico para o manejo preciso das áreas agrícolas, qualquer que seja sua escala.

Experimentos de campo são, em sua maioria, divididos em parcelas ou áreas relativamente pequenas amostradas al eatoriamente. Contudo, ao considerar as parcelas experimentais uniformes quanto aos seus atributos, mesmo em pequenas áreas, pode-se interpretar erroneamente as respostas obtidas às questões existentes, pois a hipótese de ocorrência de dependência espacial estará sendo ignorada.

A variabilidadeespacial do sol ofoi verificada em áreas de dimensões variadas, 150 ha por Biggar \& Nielsen (1976), $5.000 \mathrm{~m}^{2}$ por Lima \& Silans (1999) e mesmo em áreas menores do que $100 \mathrm{~m}^{2}$ por Reichardt et al. (1978). As propriedades químicas de uma parcela de $900 \mathrm{~m}^{2}$, a mesma utilizada para coleta de dados nestetrabal ho, foram anal isadas por Vieira (1997) que detectou variabilidade suficiente para concluir que amostragem ao acaso provavel mente esconderia a realidade.

A análise geoestatística que permite detectar a existência da variabilidade e distribuição espacial das medidas estudadas constitui importante ferramenta na análise e descrição detalhada da variabilidade das propriedades do solo (Vieira, 2000; Carvalho et al., 2002; Vieira et al., 2002). Conhecendo as coordenadas geográficas do ponto amostrado, podem-se analisar os dados, possibilitando, segundo Vendrusculo (2001), representar a área com maior detalhamento. O nível de detalhamento é obtido por meio da distância entre pontos de amostragem e depende tanto da propriedade a ser analisada quanto da escala de trabalho (tamanho da área amostrada).

As propriedades físicas e hídricas do solo estudadas por diversos autores (Es et al., 1991; Vieira et al., 1992; Nielsen et al., 1993; Sousa et al., 1999; Fietz et al.,1999; Souza et al., 2001; J oaquim J unior et al., 2002) variaram de um local para outro, apresentando continuidade ou dependência espacial, dependendo do manejo adotado e das próprias características de origem dos sol os. SegundoBeutler et al. (2002), as propriedades hídricas, como a curva de retenção de água, dependem do tipo de solo, histórico de uso e preparo do solo. Sol os manejados por diferentes preparos são alterados em profundidadee, principal mente neste caso, segundo Carvalho et al. (2002), a profundidade é um fator importante no estudo de dependência espacial.

O objetivo destetrabalho foi avaliar a variabilidade espacial de propriedades físico-hídricas do solo em uma parcela experimental, usando métodos geoestatísticos para análise de dados.

\section{MATERIAL E MÉTODOS}

O experimento foi realizado em 1982 no Centro Experimental do Instituto Agronômi co em Campinas (SP), em um Latossolo Vermel ho (E mbrapa, 1999) sob preparo convencional. Marcou-se uma parcela 
de $900 \mathrm{~m}^{2}$, com declividade de $5 \%$, para amostragem num reticulado quadrado de 49 pontos distanciados de $5 \times 5 \mathrm{~m}$. Em cada ponto foram coletadas amostras de solo deformadas e não deformadas para determinações em laboratório nas camadas de 0-25 cm e 25-50 cm de profundidade.

O teor gravimétrico de água do sol o foi obtido de acordo com Kiehl (1979), em amostras deformadas de solo seco em estufa. A resistência à penetração foi avaliada por meio de um penetrômetro, seguindo o método recomendado pela ASAE (1977), tendo sido obtidos os valores de resistência à penetração e a profundidade do sol o atingi da pel o equipamento no momento da medição.

A densidade do solo foi obtida conforme Kiehl (1979), col etando-se amostras de sol o indeformadas, com anéis vol umétricos de $100 \mathrm{~cm}^{3}$, nas profundidades de 15, 25 e $45 \mathrm{~cm}$. Estas amostras indeformadas foram submetidas às tensões de 0,$01 ; 0,03 ; 0,05 ; 0,1$; 0,$5 ; 0,8 ; 1,5 \mathrm{MPa}$, de acordo com método citado por Kiehl (1979), obtendo-se valores de retenção deágua volumétrica $\left(\mathrm{m}^{3} \mathrm{~m}^{-3}\right)$ para cada tensão, os quais foram ajustados ao modelo de Van Genuchten (1980):

$$
\theta=\theta_{\mathrm{r}}+\frac{\theta_{\mathrm{S}}-\theta_{\mathrm{r}}}{\left(\left(1+\alpha \mathrm{h}^{\mathrm{n}}\right)^{\mathrm{m}}\right)}
$$

em que $\theta$ é o teor de água no solo $\left(\mathrm{m}^{3} \mathrm{~m}^{-3}\right)$, $\theta \mathrm{r}$ e $\theta$ s são os teores deágua residual edesaturação $\left(\mathrm{m}^{3} \mathrm{~m}^{-3}\right)$, h é a tensão (MPa) aplicada em coluna de água, $\alpha, \mathrm{m}$ e $\mathrm{n}$ são parâmetros de ajuste do modelo. A água disponível $A D\left(\mathrm{~m}^{3} \mathrm{~m}^{-3}\right)$, segundo Reichardt (1987), é obtida por diferença entre o teor de água do solo na capacidade de campo $\left(\theta_{\mathrm{cc}}\right)$ e no ponto de murcha permanente $\left(\theta_{\text {pmp }}\right)$, conformea equação 2 . Para sol os tropicais, a tensão aplicada em laboratório para a capacidade de campo é de 0,01 MPa.

$$
A D=\theta_{\text {cc }}(0,01 \mathrm{MPa})-\theta_{\mathrm{pmp}}(1,5 \mathrm{MPa})
$$

I nicial mente, os dados foram analisados usando cálcul os da estatística descritiva, segundo Vieira et al. (2002), obtendo-se média, variância, coeficiente de variação, assimetria e curtose, para se ter uma visão geral de como se comportavam os dados. Também foi aplicado o teste T de significância da correlação entre cada par de variáveis, de acordo com Snedecor \& Cochran (1967).

Para verificar a dependência espacial das variáveis, interpolar dados e construir mapas, foi empregada a análise geoestatística, segundo Vieira (2000). Foram construídos semivariogramas, partindo das pressuposições de estacionaridade da hipóteseintrínseca e do cál culo da semi variância $\gamma(\mathrm{h})$ estimada na equação 3:

$$
\gamma(\mathrm{h})-\frac{1}{2 N(h)} \sum_{i=1}^{N}\left[Z\left(x_{i}\right)-Z\left(x_{i}+h\right)\right]^{2}
$$

em que $\mathrm{N}$ ( $h$ ) éo número de pares dos valores medidos $Z\left(x_{i}\right), Z\left(x_{i}+h\right)$, separados por um vetor $h$. É esperado, segundo Vieira (2000), que medições localizadas próximas sejam mais parecidas entresi do que aquelas separadas por grandes distâncias, isto é, que aumente $\gamma(\mathrm{h})$ com a distância $\mathrm{h}$ até um valor máximo, no qual se estabiliza em um patamar correspondente à distância limite de dependência espacial, que é o alcance. Medições localizadas a distâncias maiores que o al cance terão distribuição al eatória, razão por que serão independentes entre si.

Os semi variogramas que apresentaram dependência espacial foram ajustados com o model o matemático esférico (Equação 4). Os programas computacionais e procedimentos para construção e ajuste do modelo do semivariograma obedeceram às instruções de Vieira et al. (2002).

$$
\begin{gathered}
\gamma(\mathrm{h})=\mathrm{C}_{0}+\mathrm{C}_{1}\left[\frac{3}{2}\left(\frac{\mathrm{h}}{\mathrm{a}}\right)^{3}-\frac{1}{2}\left(\frac{\mathrm{h}}{\mathrm{a}}\right)^{3}\right] ; 0<\mathrm{h}<\mathrm{a} \quad \text { ou } \\
\gamma(\mathrm{h})=\mathrm{C}_{0}+\mathrm{C}_{1} ; \mathrm{h} \geq \mathrm{a}
\end{gathered}
$$

em que foram definidos os parâmetros do semivariograma, a saber: $C_{0}=$ efeito pepita que é o valor de $\gamma(h)$ quando $h=0 ; a=$ alcance, que é a distância em que $\gamma(\mathrm{h})$ permanece aproximadamente constante após aumentar com o aumento de $\mathrm{h} ; \mathrm{C}_{0}+$ $\mathrm{C}_{1}=$ patamar queéo valor de $\gamma(\mathrm{h})$ a partir do alcance e que se aproxima da variância dos dados, e $C_{1}=$ variância estrutural, ou seja, a diferença entre o patamar e o efeito pepita.

Foi calculada a razão de dependência espacial (RD), que é a proporção em percentagem do efeito pepita $\left(C_{0}\right)$ em rel ação ao patamar $\left(C_{0}+C_{1}\right)$, equação 5, e que, de acordo com Cambardella et al. (1994), apresenta a seguinte proporção: (a) dependência forte < $25 \%$; (b) dependência moderada de 26 a $75 \%$, e (c) dependência fraca $>75 \%$.

$$
R D=\left(\frac{C_{0}}{C_{0}+C_{1}}\right) \cdot 100
$$

Uma vez que o semivariograma representa a variabilidade espacial dos dados, a análise geoestatística permitiu, primeiramente, a análise dos dados, comparando-se os parâmetros de ajuste dos semivariogramas $\left(\mathrm{C}_{0}, \mathrm{C}_{1}\right.$, a e RD) para cada uma das variáveis (teor deágua, resistência à penetração, densidade do solo, coeficientes e água disponível).

Observada a existência de dependência espacial definida pelo semivariograma, os valores vizinhos serão tão semel hantes que haverá possibilidade de estimar valores para qual quer local onde a variável não foi medida, usando-se a krigagem, segundo Vieira et al. (2002), que estima valores com as condições de estimativa sem tendenciosidade e com desvios míni mos em relação aos val ores conhecidos, ou seja, com variância mínima (E quação 6): 


$$
\sum_{j=1}^{N} \lambda_{j} \gamma\left(x_{i}, x_{j}\right)_{+\mu}=\gamma\left(x_{i}, x_{0}\right), i=1, N \quad \sum_{j=1}^{N} \lambda_{j}=1
$$

em que $\gamma\left(x_{i}, x_{j}\right)$ é a semivariância estimada, usando - modelo ajustado ao semivariograma, correspondente à distância entre os pontos localizados na posição $x_{i}$ e $x_{j}$ e $\gamma\left(x_{i}, x_{0}\right)$ é a semivariância correspondente à distância entre os pontos localizados na posição $x_{i}$ ex $_{0}$. Valores de peso $\lambda$ e um valor do mutiplicador de Lagrange, $\mu$, associado com a minimização da variância, são gerados e com os valores de $\lambda_{i}$ podem-se estimar valores (Z) no espaço amostrado para qualquer posição $x_{0}$. Com os val ores estimados (E quação 7), foram construídos mapas de isolinhas, em função da coordenada geográfica. O uso da Krigagem para mostrar a variabilidade espacial interpola valores para que sejam construídos mapas de isol inhas.

$$
\mathrm{Z}\left(\mathrm{x}_{\mathrm{o}}\right)=\sum_{\mathrm{i}=1}^{\mathrm{N}} \lambda_{\mathrm{i}} \mathrm{Z}(\mathrm{xi})
$$

As construções dos mapas com os val ores obti dos por meio da krigagem são importantes para a verificação e interpretação da variabilidade espacial. A anál ise geoestatística dos dados écompl etada com as informações mostradas nos mapas visualmente comparadas para o entendimento da variabilidade das propriedades físicas e hídricas do sol o no campo e que são úteis nas tomadas de decisões.

\section{RESULTADOS E DISCUSSÃO}

Os resultados estatísticos das variáveis do solo analisadas estão nos quadros 1 e 2, enquanto os parâmetros do semivariograma das variáveis que apresentaram dependência espacial estão no quadro 3. As variáveis, em sua maioria, apresentaram assimetria e curtose compatíveis com a distribuição normal (valores próximos de 0) e coeficientes de variação de médios a baixos (<20). O $\alpha$ em 0-25 e $25-50 \mathrm{~cm}$ de profundidade apresentou os maiores valores para coeficiente de variação, considerado alto (>20), seguido da água disponível para as duas profundidades, segundo a classificação adotada por Carvalho et al. (2002). Considerando o tamanho da parcela, os coeficientes de variação demostram a importância de se amostrar intensamente mesmo em áreas pequenas, estando de acordo com os coeficientes encontrados por Vieira (1997).

Observa-se, também ( Quadro 1), quea média da resistência à penetração apresenta-se consideravelmente alta, equiparando-se aos val ores encontrados por Rosolem et al. (1994), Araújo et al . (1999) e Grego \& Benez (1999), obtidos em solos argilosos sob sistema de plantio direto com compactação na camada superficial do solo. A resistência à penetração encontrada pode estar associada à compactação de subsuperfície que geralmente ocorre no preparo convencional; no entanto, não se sabe em que profundidade isto ocorreu, pois ovalor disponível éum

Quadro 1. Parâmetros estatísticos da profundidade atingida pelo penetrômetro, da resistência média à

\begin{tabular}{|c|c|c|c|c|c|}
\hline Variável & Média & Variância & CV (\%) & Assimetria & Curtose \\
\hline Profundidade do penetrômetro $(\mathrm{cm})$ & 52,92 & 51,70 & 13,60 & 0,42 & 0,07 \\
\hline \multirow[t]{2}{*}{ Resistência média à penetração ( $\mathrm{MPa}$ ) } & 2,75 & 0,21 & 16,88 & 0,04 & 0,20 \\
\hline & \multicolumn{5}{|c|}{ Profundidade de $0-25 \mathrm{~cm}$} \\
\hline Teor gravimétrico de água ( $\left.\mathrm{g} \mathrm{g}^{-1}\right)$ & 0,25 & $2,00 \times 10^{-4}$ & 6,05 & $-0,70$ & 0,74 \\
\hline$\theta r\left(m^{3} m^{-3}\right)$ & 0,25 & $0,05 \times 10^{-2}$ & 8,99 & 0,33 & 0,13 \\
\hline$\theta s\left(m^{3} m^{-3}\right)$ & 0,53 & $0,09 \times 10^{-2}$ & 5,79 & 0,68 & 0,38 \\
\hline Parâmetro $\alpha$ & 0,02 & $0,02 \times 10^{-2}$ & 95,20 & 2,97 & 10,71 \\
\hline Parâmetro m & 0,49 & $0,07 \times 10^{-2}$ & 16,99 & $-0,10$ & 0,19 \\
\hline Parâmetro n & 2,02 & 0,13 & 17,67 & 0,99 & 1,32 \\
\hline Retenção água 0,01 M pa $\left(\mathrm{m}^{3} \mathrm{~m}^{-3}\right)$ & 0,45 & $0,41 \times 10^{-2}$ & 14,10 & 0,37 & $-0,19$ \\
\hline Retenção água 0,03 M pa $\left(\mathrm{m}^{3} \mathrm{~m}^{-3}\right)$ & 0,35 & $0,02 \times 10^{-2}$ & 12,42 & 0,58 & $-0,01$ \\
\hline Retenção água $0,05 \mathrm{Mpa}\left(\mathrm{m}^{3} \mathrm{~m}^{-3}\right)$ & 0,29 & $0,13 \times 10^{-2}$ & 11,89 & 0,09 & $-0,55$ \\
\hline Retenção água $0,1 \mathrm{MPa}\left(\mathrm{m}^{3} \mathrm{~m}^{-3}\right)$ & 0,28 & $0,08 \times 10^{-2}$ & 10,27 & 0,75 & 0,61 \\
\hline Retenção água $0,5 \mathrm{MPa}\left(\mathrm{m}^{3} \mathrm{~m}^{-3}\right)$ & 0,25 & $0,05 \times 10^{-2}$ & 9,72 & 0,92 & 1,35 \\
\hline Retenção água $0,8 \mathrm{MPa}\left(\mathrm{m}^{3} \mathrm{~m}^{-3}\right)$ & 0,25 & $0,07 \times 10^{-2}$ & 10,92 & 0,38 & 0,03 \\
\hline Retenção água $1,5 \mathrm{MPa}\left(\mathrm{m}^{3} \mathrm{~m}^{-3}\right)$ & 0,25 & $0,05 \times 10^{-2}$ & 9,29 & 0,30 & $-0,07$ \\
\hline Água disponível $\left(\mathrm{m}^{3} \mathrm{~m}^{-3}\right)$ & 0,20 & $0,26 \times 10^{-2}$ & 25,18 & $-0,00014$ & 0,0052 \\
\hline Densidade a $15 \mathrm{~cm}\left(\mathrm{~g} \mathrm{~cm}^{-3}\right)$ & 1,33 & 0,02 & 10,89 & 0,47 & 0,45 \\
\hline Densidade a $25 \mathrm{~cm}\left(\mathrm{~g} \mathrm{~cm}^{-3}\right)$ & 1,31 & 0,02 & 9,65 & 0,66 & 0,35 \\
\hline
\end{tabular}
penetração e das variáveis amostradas a 0-25 cm de profundidade

$\mathrm{CV}(\%)=$ coeficiente de variação. 


\section{Quadro 2. Parâmetros estatísticos das variáveis amostradas a $\mathbf{2 5 - 5 0} \mathrm{cm}$ de profundidade}

\begin{tabular}{|c|c|c|c|c|c|}
\hline Variável & Média & Variância & CV (\%) & Assimetria & Curtose \\
\hline Teor gravimétrico de água ( $\left.\mathrm{g} \mathrm{g}^{-1}\right)$ & 0,26 & $1,00 \times 10^{-4}$ & 3,87 & 0,39 & 1,59 \\
\hline$\theta r\left(m^{3} m^{-3}\right)$ & 0,25 & $0,09 \times 10^{-2}$ & 12,16 & 0,33 & $-0,73$ \\
\hline$\theta s\left(m^{3} m^{-3}\right)$ & 0,52 & $0,22 \times 10^{-2}$ & 9,01 & $-0,37$ & $-0,85$ \\
\hline Parâmetro $\alpha$ & 0,04 & $0,83 \times 10^{-3}$ & 78,66 & 2,79 & 12,06 \\
\hline Parâmetro m & 0,36 & $0,32 \times 10^{-2}$ & 15,60 & 1,22 & 2,44 \\
\hline Parâmetro n & 2,02 & 0,13 & 17,67 & 0,99 & 1,32 \\
\hline Retenção água 0,01 MPa $\left(\mathrm{m}^{3} \mathrm{~m}^{-3}\right)$ & 0,37 & $0,21 \times 10^{-2}$ & 12,18 & 0,82 & 0,65 \\
\hline Retenção água $0,03 \mathrm{MPa}\left(\mathrm{m}^{3} \mathrm{~m}^{-3}\right)$ & 0,34 & $0,16 \times 10^{-2}$ & 11,82 & 0,49 & $-0,73$ \\
\hline Retenção água $0,05 \mathrm{MPa}\left(\mathrm{m}^{3} \mathrm{~m}^{-3}\right)$ & 0,31 & $0,12 \times 10^{-2}$ & 11,33 & 0,45 & $-0,07$ \\
\hline Retenção água $0,1 \mathrm{MPa}\left(\mathrm{m}^{3} \mathrm{~m}^{-3}\right)$ & 0,30 & $0,12 \times 10^{-2}$ & 11,58 & 0,58 & $-0,49$ \\
\hline Retenção água 0,5 MPa $\left(\mathrm{m}^{3} \mathrm{~m}^{-3}\right)$ & 0,27 & $0,94 \times 10^{-3}$ & 11,53 & 0,14 & $-0,75$ \\
\hline Retenção água 0,8 MPa $\left(\mathrm{m}^{3} \mathrm{~m}^{-3}\right)$ & 0,57 & $0,1 \times 10^{-2}$ & 12,47 & 0,49 & $-0,51$ \\
\hline Retenção água 1,5 MPa $\left(\mathrm{m}^{3} \mathrm{~m}^{-3}\right)$ & 0,27 & $0,82 \times 10^{-3}$ & 10,50 & 0,13 & $-0,53$ \\
\hline Água disponível $\left(\mathrm{m}^{3} \mathrm{~m}^{-3}\right)$ & 0,11 & $0,08 \times 10^{-2}$ & 26,24 & 0,62 & 1,67 \\
\hline Densidade a $45 \mathrm{~cm}\left(\mathrm{~g} \mathrm{~cm}^{-3}\right)$ & 1,29 & $1,84 \times 10^{-2}$ & 10,53 & 0,56 & $-0,62$ \\
\hline
\end{tabular}

$\mathrm{CV}(\%)=$ coeficiente de variação.

valor médio para uma profundidade média de $52,92 \mathrm{~cm}$.

O modelo matemático esférico ajustado a todos os semivariogramas éo que predomina nos trabalhos em ciência do solo (Vieira, 1997; Souza et al., 2001; Carvalho et al., 2002). McBratney \& Webster (1986) estudaram modelos de ajuste do semivariograma para as propriedades do solo e relataram que os modelos esférico e exponencial são os mais freqüentemente encontrados. A respeito da razão de dependência (RD), observa-se (Quadro 3), que a maioria das variáveis apresentam moderada dependência espacial (26-75\%), concordando com Souza et al. (2001) e Carvalho et al . (2002). Observase que o teor de água de 0-25 cm apresentou forte dependência $(\leq 25 \%)$. Este resultado mostra a importância da caracterização espacial desta variável mesmo em pequenas áreas, como a parcela em estudo.

A dependência espacial ocorreu principalmente nas variáveis obtidas na camada superior do solo de 0-25 cm (Quadro 3), concordando com a dependência encontrada por Vieira (1997) para propriedades químicas, e somente duas variáveis apresentaram dependência espacial na camada de $25-50 \mathrm{~cm}$. A ausência de dependência espacial nas demais variáveis foi verificada pela inexistência do patamar $\left(\mathrm{C}_{0}+\mathrm{C}_{1}\right)$ nos semivariogramas, ocorrendo o que se denomina efeito pepita puro, segundo Vieira (2000), impossibilitando, portanto, o ajuste de um modelo ao semivariograma, razão pela qual seus parâmetros não constam no quadro 3. A explicação para a ocorrência da dependência espacial na camada superficial pode estar associada ao preparo do solo convencional, onde os equipamentos de preparo, arado e grade movimentam demasiadamente a camada superior afetando sua estrutura original, tornando pontos próximos entresi mais semel hantes do que os mais distantes, bem como ao fato de ser o solo muito argiloso e bem estruturado. De acordo com Prado (2003), os Latossolos apresentam partículas bem estruturadas pedologicamente e, quando associadas ao revolvimento da camada superficial, podem ter favorecido ainda mais a dependência espacial encontrada, o que não aconteceu na camada inferior $(25-50 \mathrm{~cm})$, onde houve pouco ou nenhum revolvimento.

Os menores valores para o alcance foram para resistência à penetração, profundidade do penetrômetro e teor de água de saturação, indicando que os pontos são mais semel hantes entre si num raio de $8 \mathrm{~m}$ aproximadamente, já, para as demais variáveis, os raios aumentam na média para $13 \mathrm{~m}$ (Quadro 3). I sto mostra que o al cance da dependência espacial depende da variável e que, se as amostragens realizadas nesta área considerassem este alcance, os dados obtidos poderiam representar melhor a realidade da área amostrada. Variáveis como o teor de água podem ser amostradas em espaçamentos maiores, pois o alcance obtido foi maior do que para as demais, ao passo que medições pontuais, como a resistência à penetração, podem resultar em dependência espacial menor e devem ser amostradas em distâncias menores.

A figura 1 mostra os semivariogramas ajustados pelo modelo esférico da resistência média à penetração e do teor de água em 0-25 cm, variáveis que apresentaram alcances de 8,75 e 16,86 m, respectivamente. Vieira (1997) encontrou alcance de $20 \mathrm{~m}$ e relação de dependência de $40 \%$ para as propriedades químicas do solo, nesta mesma área, indicando que a quantidade de variação ao acaso ea 
Quadro 3. Parâmetros do modelo esférico ajustado aos semi variogramas: efeito pepita $\left(C_{0}\right)$, patamar $\left(C_{1}\right)$, alcance (a), grau de dependência (RD)

\begin{tabular}{|c|c|c|c|c|}
\hline \multirow[b]{2}{*}{ Variável } & \multirow[b]{2}{*}{ Co } & \multirow[b]{2}{*}{$\mathrm{C}_{1}$} & \multirow[b]{2}{*}{$a(m)$} & RD (\%) \\
\hline & & & & $\left(\frac{C_{0}}{C_{0}+C_{1}}\right) \times 100$ \\
\hline Profundidade do penetrômetro $(\mathrm{cm})$ & 25,00 & 31,00 & 8,00 & 44,64 \\
\hline Resistência média a penetração (MPa) & 0,13 & 0,10 & 8,75 & 56,52 \\
\hline Densidade a $15 \mathrm{~cm}\left(\mathrm{~g} \mathrm{~cm}^{-3}\right)$ & $0,12 \times 10^{-1}$ & $0,12 \times 10^{-1}$ & 11,00 & 50,00 \\
\hline \multirow[t]{2}{*}{ Densidade a $25 \mathrm{~cm}\left(\mathrm{~g} \mathrm{~cm}^{-3}\right)$} & $0,53 \times 10^{-2}$ & $0,12 \times 10^{-1}$ & 10,00 & 30,63 \\
\hline & \multicolumn{4}{|c|}{ Profundidade $0-25 \mathrm{~cm}$} \\
\hline Teor gravimétrica de água $\left(\mathrm{g} \mathrm{g}^{-1}\right)$ & 0,29 & 2,23 & 16,86 & 11,75 \\
\hline$\theta r\left(m^{3} m^{-3}\right)$ & $0,02 \times 10^{-2}$ & $0,04 \times 10^{-2}$ & 11,00 & 27,27 \\
\hline$\theta s\left(m^{3} m^{-3}\right)$ & $0,07 \times 10^{-2}$ & $0,04 \times 10^{-2}$ & 8,00 & 62,50 \\
\hline$\alpha\left(\mid \mathrm{cm}^{-1}\right)$ & $0,01 \times 10^{-2}$ & $0,15 \times 10^{-2}$ & 13,00 & 25,00 \\
\hline Coeficiente $\mathrm{m}$ (sdm) & $0,25 \times 10^{-2}$ & $0,50 \times 10^{-2}$ & 9,00 & 33,33 \\
\hline Retenção água $0,03 \mathrm{MPa}\left(\mathrm{m}^{3} \mathrm{~m}^{-3}\right)$ & $0,09 \times 10^{-2}$ & $0,09 \times 10^{-2}$ & 10,00 & 48,65 \\
\hline Retenção água $0,05 \mathrm{MPa}\left(\mathrm{m}^{3} \mathrm{~m}^{-3}\right)$ & $0,06 \times 10^{-2}$ & $0,08 \times 10^{-2}$ & 11,00 & 42,86 \\
\hline Retenção água $0,1 \mathrm{MPa}\left(\mathrm{m}^{3} \mathrm{~m}^{-3}\right)$ & $0,05 \times 10^{-2}$ & $0,04 \times 10^{-2}$ & 13,00 & 55,00 \\
\hline Retenção água $0,5 \mathrm{MPa}\left(\mathrm{m}^{3} \mathrm{~m}^{-3}\right)$ & $0,03 \times 10^{-2}$ & $0,03 \times 10^{-2}$ & 14,00 & 50,72 \\
\hline Retenção água $0,8 \mathrm{MPa}\left(\mathrm{m}^{3} \mathrm{~m}^{-3}\right)$ & $0,03 \times 10^{-2}$ & $0,04 \times 10^{-2}$ & 11,00 & 45,94 \\
\hline \multirow[t]{2}{*}{ Retenção água $1,5 \mathrm{MPa}\left(\mathrm{m}^{3} \mathrm{~m}^{-3}\right)$} & $0,03 \times 10^{-2}$ & $0,03 \times 10^{-2}$ & 10,00 & 50,00 \\
\hline & \multicolumn{4}{|c|}{ Profundidade $25-50 \mathrm{~cm}$} \\
\hline Teor água gravimétrica ( $\mathrm{g} \mathrm{g}^{-1}$ ) & 0,60 & 0,50 & 20,00 & 54,54 \\
\hline Água disponível $\left(\mathrm{m}^{3} \mathrm{~m}^{-3}\right)$ & $0,05 \times 10^{-2}$ & $0,04 \times 10^{-2}$ & 19,00 & 55,55 \\
\hline
\end{tabular}

distância entre vizinhos são semelhantes às encontradas para as características físicas analisadas neste trabalho.

O testeT designificância da correlação entre cada par de variáveis (Snedecor \& Cochran, 1967) resultou na correlação positiva entre a densidade do solo a 15 e

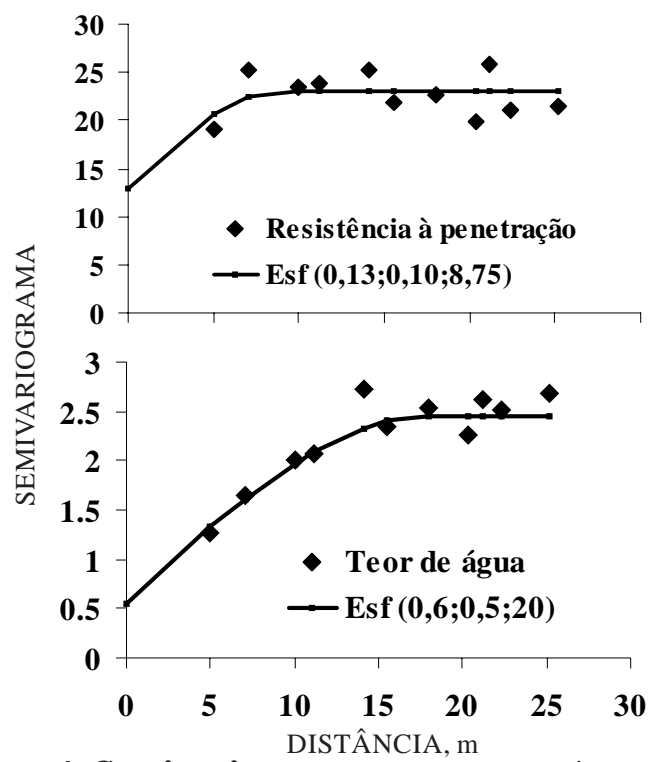

Figura 1. Semivariogramas com os parâmetros de ajuste esférico (E ff $\left(\mathrm{C}_{0} ; \mathrm{C}_{1} ; \mathrm{a}\right)$ ) para resistência à penetração $(\mathrm{MPa})$ e teor de água $\left(\mathrm{g} \mathrm{g}^{-1}\right)$ de 0-25 cm de profundidade.
$25 \mathrm{~cm}$ de profundidade e as retenções de água de 0 $25 \mathrm{~cm}$, com exceção da retenção de água na tensão de 0,01 MPa (Figura 2).

Houve correl ação positiva entre retenção de água e densidade do solo, pois o solo argiloso tem naturalmente grande capacidade de reter água, graças à microporosidadeemaior agregação existente (Kiehl, 1979). Apesar de não estudar a variabilidade espacial, Beutler et al. (2002) também encontraram correlação positiva para retenção deágua edensi dade do sol o no sistema de cultivo de cana-de-açúcar.

As figuras $3 a$ a 30 mostram os mapas das variáveis com valores estimados por krigagem agrupados numa escala de cinco classes, após a

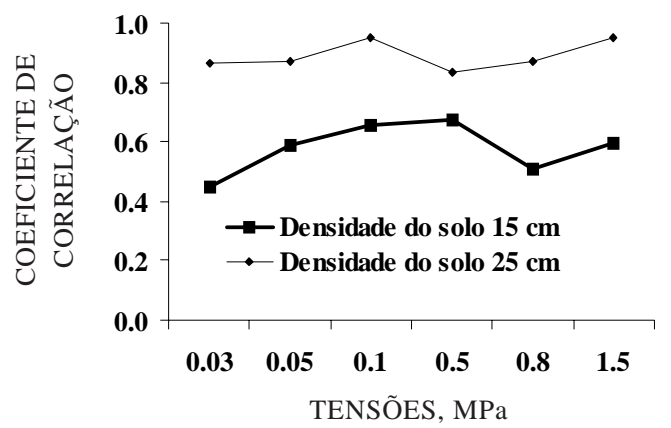

Figura 2. Coeficiente de correlação entre a densidade do solo $\left(\mathrm{g} \mathrm{cm}^{-3}\right)$ a 15 e $25 \mathrm{~cm}$ de profundidade e a retenção de água $\left(\mathrm{m}^{3} \mathrm{~m}^{-3}\right)$ nas seis tensões (MPa). 
análise de dependência espacial verificada nos semivariogramas. Fez-sea inter pretação dos mapas visualmente, onde a direção do declive da área se dá com o decréscimo da distância y.

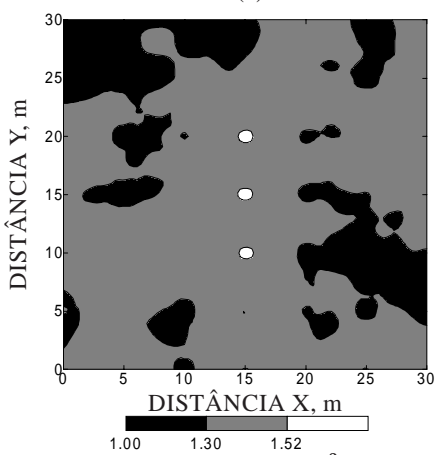

Densidade do solo $\mathrm{g} \mathrm{cm}^{-3}$ a $15 \mathrm{~cm}$

(e)

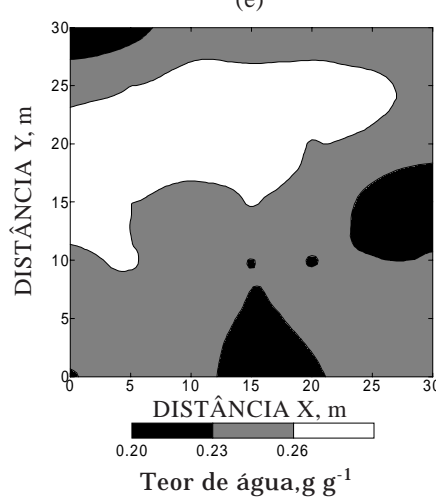

$0-25 \mathrm{~cm}$

(i)

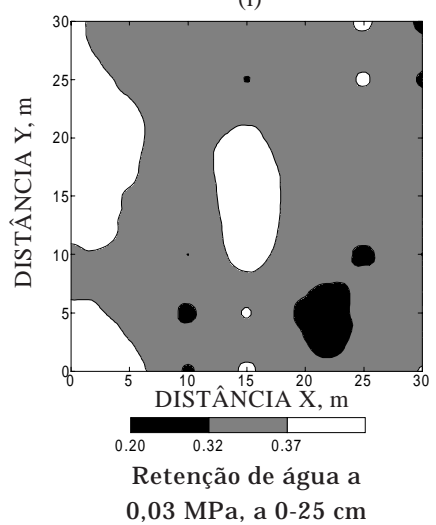

(m)

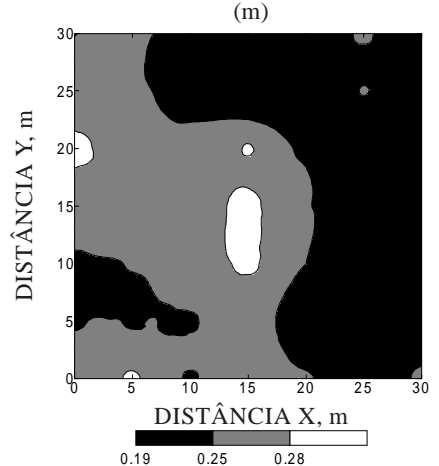

Retenção de água a 0,8 MPa, a 0-25 cm (b)

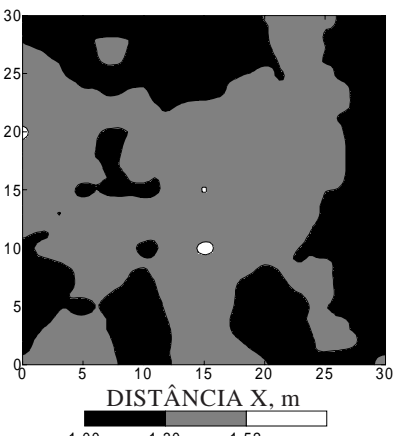

(f)

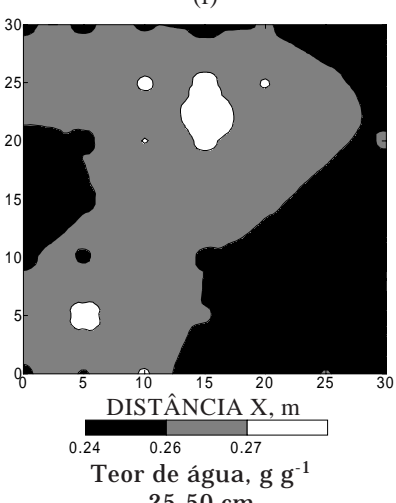

$25-50 \mathrm{~cm}$

(j)

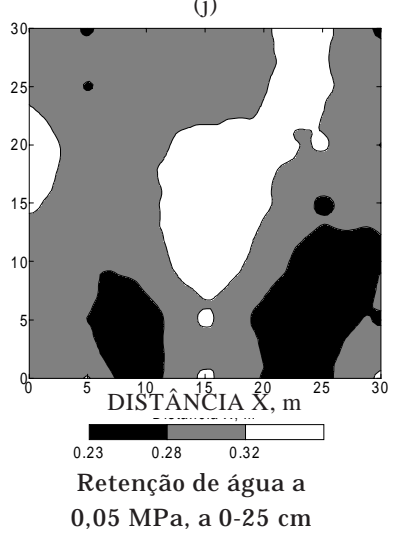

(c)

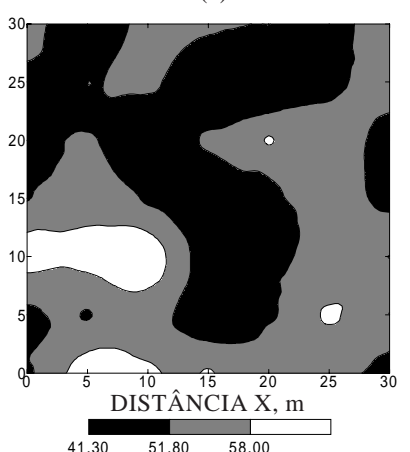

(g)

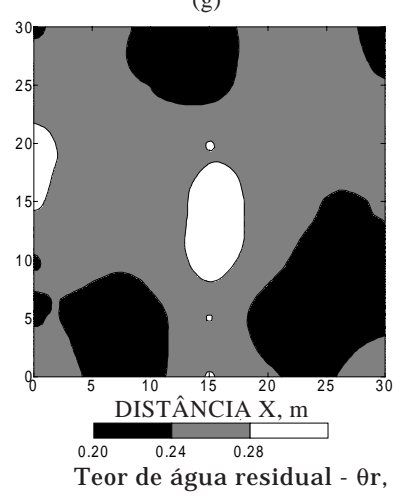

$\mathrm{m}^{3} \mathrm{~m}^{-3}$ a $0-25 \mathrm{~cm}$

(k)

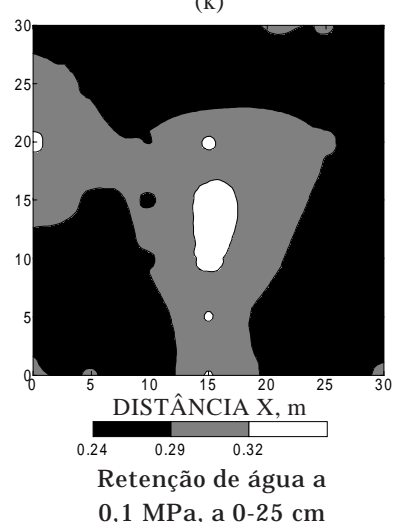

(n)

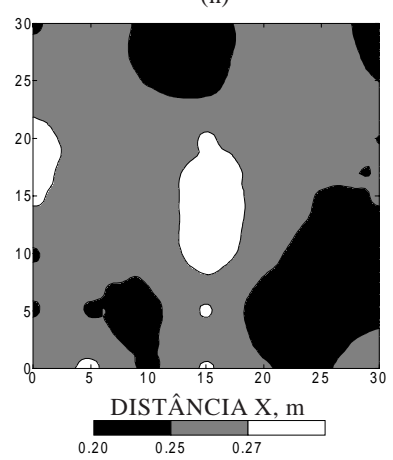

Retenção de água a 1,5 MPa, a 0-25cm (d)

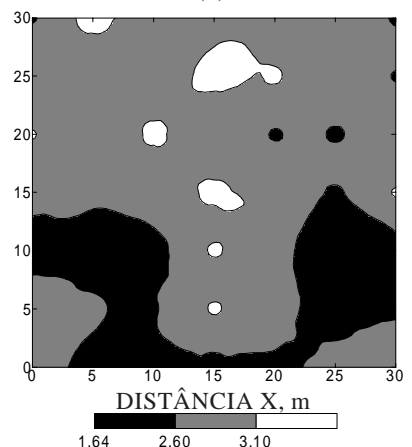

Resistência à penetração, MPa

(h)

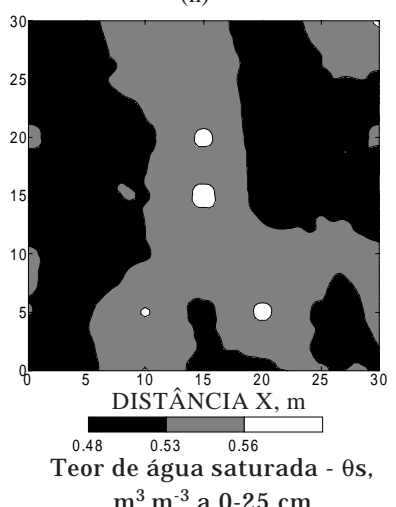

(1)

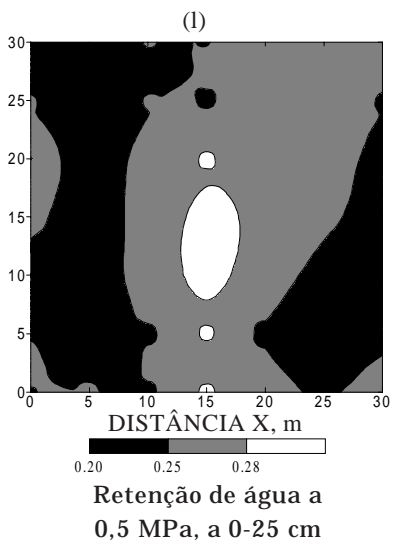

(o)

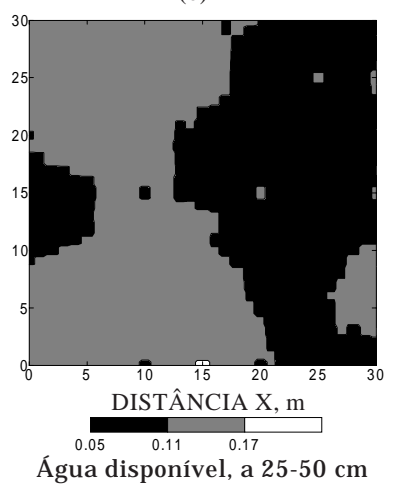

Figura 3. Mapas das variáveis estudadas, a diferentes profundidades, com valores estimados por krigagem. 
As figuras $3 a$ e b mostram que as variabilidades das densidades do solo de $0-25 \mathrm{~cm}$ e de $25-50 \mathrm{~cm}$ de profundidade são bastante semelhantes, e a correlação positiva com a retenção de água nas seis tensões (Figuras 3i, j, k, l, m e n) pode ser verificada pela semelhança das manchas com maiores valores na parte central da parcela. No entanto, não segue o comportamento da resistência média à penetração, que apresentou nitidamente os maiores valores à medida que aumenta a distância y (Figura 3d), indicando quea variabilidade da área adensada pode ser, neste caso, mais bem representada pela resistência à penetração do que pela densidade do solo, que apresentou manchas mais dispersas.

A profundidademédia atingi da pel o penetrômetro (Figura 3c) apresentou comportamento contrário à resistência (Figura $3 d$ ), uma vez que onde o solo oferece maior resistência à penetração, parte superior da área, a profundidade atingida pelo penetrômetro é menor. J á os teores gravimétricos de água (Figuras 3e, f) do solo apresentaram-se menores na parte inferior direita da ár ea e parecem não ter relação com os resultados da profundidade do penetrômetro e da resistência à penetração.

Na maioria dos mapas dos teores deágua residual (Figura $3 g$ ), de saturação (Figura 3h) e retenção de água (Figuras $3 i, j, k$, l, m e n), existem áreas com valores maiores na direção do declive e na região central da área. Estas áreas indicam que esta parte da parcela émais densa, oferece mai or resistência e representa maior teor e retenção de água. Entretanto, à medida que se distância para a direção $x$, observa-se um comportamento também semel hante para as variáveis, e os val ores tendem a diminuir. Este resultado reforça ainda mais a variabilidade encontrada nesta parcela por Vieira (1997) para as propriedades quími cas do solo, apesar de ser considerada uma área relativamente pequena, ficando claro que não é o tamanho da parcela que determina seu grau de homogeneidade.

A existência de dependência espacial das variáveis, bem comoa semelhança de comportamento entre elas, sugere que se analise a variabilidade espacial das propriedades do sol o em conjunto, antes de adotar um delineamento experimental ao acaso, visto que qual quer tratamento adotado nesta parcela que necessite da homogeneidade levará a falsos resultados.

\section{CONCLUSÕES}

1. A parcela experimental apresentou variabilidade espacial com razão de dependência moderada para a maioria das variáveis, principalmente na camada superficial $(0-25 \mathrm{~cm})$ eal cance variando de 8 a $20 \mathrm{~m}$.

2. A região central da parcela, na direção do declive, apresentou maior densidade do solo, maior resistência à penetração e maior teor e retenção de água.
3. A dependência espacial encontrada para as variáveis, bem como a semel hança decomportamento entreelas, permiteinferir que amostragem ao acaso resultaria em interpretações incorretas efal has, pois esconderia a variabilidade encontrada na área amostrada, pois não foi o tamanho que determinou seu grau de homogeneidade.

\section{LITE RATURA CITADA}

AMERICAN SOCIETY AGRICULTURAL OF CONGINERING - ASAE. Recommendation ASAE R.313.1 Soil cone penetrometer. Agricultural Engineers Year Book, 1977. p.347-348.

ARAÚJ O, A.G.; CASÃO JUNIOR, R.; RALISCH, R. \& SIQUEIRA, R. Mobilização de solo e emergência de plantas na semeadura direta de soja (Glycinemax L.) e milho (Zea mays L.) em solos argilosos. Eng. Agríc., 19:226-237, 1999.

BEUTLER, A.N.; CENTURION, J .F.; SOUZA, Z.M.; ANDRIOLI, I. \& ROQUE, C.G. Retenção de água em dois tipos de Latossolo sob diferentes usos. R. Bras. Ci. Solo, 26:829834, 2002.

BIGGAR, J .N.; \& NIELSEN, D.R. The spatial variability of the leaching characteristics of a field soil. Water Res. Res., 14:263-271, 1976.

CAMBARDELLA, C.A.; MOOMAN, T.B.; NOVAK, J.M.; PARKIN, T.B.; KARLEM, D.L.; TURVO, R.F.\& KONOPA, A.E. Field scale variability of soil properties in central I owa soil. Soil Sci. Am. J ., 47:1501-1511, 1994.

CARVALHO, J.R.P.; SILVEIRA, P.M. \& VIEIRA, S.R. Geoestatística na determinação da variabilidade espacial de características químicas do solo sob diferentes preparos. Pesq. Agropec. Bras., 37:1151-1159, 2002.

EMPRESA BRASILEIRA DE PESQUISA AGROPECUÁRIA EMBRAPA. Sistema brasileiro de classificação de solos. Brasília, Embrapa Produção del nformações; Rio deJ aneiro: Embrapa Solos, 1999. 412p.

ES, H.M.V.; CASSEL, D.K. \& DANIELS, R.B. Infiltration variability and correlations with surface soil properties for eroded Hapludult. Soil Sci. Soc. Am. J ., 55:486-492, 1991.

FIETZ, C.R.; FOLEGATTI, M.V.; VIEIRA, S.R. \& FRIZZONE, J.A. Efeito da variabilidade do armazenamento de água no solo na qualidade da irrigação por aspersão. R. Bras. Eng. Agríc. Amb., 3:150-153, 1999.

GREGO, C.R. \& BENEZ, S.H. Cobertura vegetal espontânea e produtividade da cultura do feijão (Phaseol us vulgaris L.), através do preparo do solo e manejo da cobertura. R. Energia Agric., 14:29-38, 1999.

J OAQUIM J UNIOR, G.O.; CICHOTA, R.; CONDINI, F.G.M. \& van LIER, Q.J. Padrões de variabilidade espacial para densidade e umi dade em um Latossolo Vermel ho-Amarelo álico. In: REUNIÃO BRASILEIRA DE MANEJO E CONSERVAÇÃO DO SOLO E DA ÁGUA, 14., Cuiabá, 2002. CD-ROM

KIEHL, E.J . Manual de edafologia: relação solo-água-planta. São Paulo, Agronômica Ceres, 1979. 262p. 
LIMA, C.A.G. \& SILANS, A.P. Variabilidade espacial da infiltração de água no solo. Pesq. Agropec. Bras., 34:23112320, 1999

MC BRATNEY,A.B. \& WEBSTER, R. Choosing functions for semi-variograms of soil properties and fitting them to sampling estimates. J. Soil Sci., 37:617-639, 1986.

NIELSEN, D.R.; TOLLOTSON, P.M. \& VIEIRA, S.R. Analysing field-measured soil-water properties. Agric. Water Manag., 6:93-109, 1983.

PRADO, H. Solos do Brasil-Gênese, morfologia, levantamento, manejo agrícola e geotécnico. 3.ed. Piracicaba, Escola Superior de Agricultura "Luiz de Queiroz", 2003. 275p.

REICHARDT, K. A água em sistemas agrícolas. São Paulo, Manole, 1987. 188p.

REICHARDT, K.; GROHMANN, F.; LIBARDI, P.L. \& NASCIMENTO, V.F. Condutividade hidráulica saturada de um perfil de Terra Roxa estruturada (Alfisol). R. Bras. Ci. Solo, 2:21-24, 1978.

ROSOLEM, C.A.; ALMEIDA, A.C.S. \& SACRAMENTO, L.V.S. Sistema radicular e nutrição da soja em função da compactação do solo. Bragantia, 53:259-266, 1994.

SNEDECOR, G.W. \& COCHRAN, W.G. Statistical methods. 6.ed. Ames, 1967. p.172-195.

SOUSA, J .R.; QUEIROZ, J .E. \& GHEYI, H.R. Variabilidade espacial de características físico-hídricas e de água disponível em um solo aluvial no semi-árido paraibano. R. Bras. Eng. Agríc. Ambiental, 3:140-144, 1999.
SOUZA, M.Z.; SILVA, M.L.S.; GUIMARÃES, G.L.; AMPOS, D.T.S.; CARVALHO, M.P. \& PEREIRA, G.T. Variabilidade espacial de atributos físicos em um Latossolo Vermelho distrófico sob semeadura direta em Selvíria (MS). R. Bras. Ci. Solo, 25:699-707, 2001.

van GENUCHTEN, M.T. A closed-form equation for predicting the hydraulic conductivity of unsaturated soils. Soil Sci. Soc. Am. J ., 44:892-8, 1980.

VENDRUSCULO, L.G. Desenvolvimento de um sistema computacional para análise geoestatística. Campinas, Universidade de Campinas, 2001, 87p. (Tese de Mestrado)

VIEIRA, S.R. Geoestatística em estudos de variabilidade espacial do solo. In: NOVAIS, R.F.; ALVAREZ V., V.H. \& SCHAEFER, G.R., eds. Tópicos em ciência do solo. Viçosa, Sociedade Brasileira de Ciência do Solo, 2000. v.1, p.1-54.

VIEIRA, S.R. Variabilidade espacial de argila, silte e atributos químicos em uma parcela experimental de um Latossolo Roxo de Campinas, SP. Bragantia, 56:181-190, 1997.

VIEIRA, S.R.; DE MARIA, I.C.; LOMBARDI NETO, F.; DECHEN, S.C.F. \& CASTRO, O.M. Caracterização da variabilidade espacial de propriedades físicas. Campinas, 1992. p.41-51 (Documentos IAC, 29)

VIEIRA, S.R.; MILLETE, J .; TOPP, G.C. \& REYNOLDS, W.D. Handbook for geostatistical analysis of variability in soil and climate data. In: ALVAREZ V., V.H.; SCHAEFER, C.E.G.R.; BARROS, N.F .; MELLO, J .W.V. \& COSTA, L.M., eds. Tópicos em ciência do solo. Viçosa, Sociedade Brasileira de Ciência do Solo, 2002. v.2, p.1-45. 
